\title{
AN ASSESSMENT MODEL FOR ENTERPRISE ARCHITECTURE IMPLEMENTATION IN PUBLIC SECTOR ORGANISATION
}

\begin{abstract}
Despite of many Enterprise Architecture (EA) frameworks and methodologies available, in reality EA implementation is a challenging process. In order to assure a progressive EA implementation, assessment and monitoring mechanism is required. The existing EA assessment approaches are mostly based on checklist or maturity model and design to assess post EA implementation. Less EA assessment is found to cater on the pre and during EA implementation process. This indicates that, lack of systematic assessment mechanism exist especially for pre and during EA implementation phase. Hence, based on the gap identified, this study proposes a priority based assessment model during EA implementation process. This model was tested in three selected case studies and the results indicate this model is well function and accepted by the user.
\end{abstract}

Keywords: Enterprise Architecture (EA), Analytic Hierarchy Process (AHP), Balanced Scorecard

\section{Introduction}

Recently, Enterprise Architecture (EA) is gaining the attention from the public sector as a solution to improve the function of e-Government. EA is a hierarchical approach for aligning business and Information Technology (IT) by integrating the information systems, processes, organisational units and people in an organisation. Despite of many EA frameworks and methodologies available, in reality EA implementation is a challenging process. This is due to inflexibility and complexity of the unique business function and information technology structures of the organisation itself. In order to assure a progressive EA implementation, assessment and monitoring mechanism is required. Therefore, the aim of this research is to develop a priority based assessment model that is able to provide specific results according to the organisation requirements and conditions during EA implementation process in the context of public sector organisation.

\section{Literature Review}

Specific measurement and method makes it possible to assess the legitimacy, efficiency, stakeholder satisfaction and EA value of the organisation (Rodrigues and Amaral, 2010). However, the existing EA assessments are mostly based on sequence checklist and maturity model approach, which are very generic in nature (Bakar et al., 2015; Sobczak, 2013). In addition, most of the current assessment models were designed to assess the post EA implementation process without specifying any type of organisation and the business nature (Pruijt et al., 2012; Van der Raadt et al., 2010). Hence, there is none of assessment model available for EA implementation process tailored according to priority of business nature of that organisation especially for public sector organisation.

\section{Hypotheses/Objectives}

The aim of this model is to assess the public sector organisation priority in implementing EA. Based on BSC perspectives and findings from case study, this paper proposed six assessment categories, which are Internal Process, Learning and Growth, Authority Support, Cost, Technology and Talent Management. Within these categories, there are 27 assessment criteria used for this model.

\section{Research Design/Methodology}

This model is developed based on Balanced Scorecard (BSC) and Analytic Hierarchy Process (AHP). Main data were gathered from multiple case studies with the scope of Malaysian Public Sector agencies that have implemented EA. Data were collected through interviews with the agencies EA team, general observation during the EA workshops as well as review of EA related documents. The case studies finding is then formulated as a set of assessment criteria embed with pairwise comparison calculation based on AHP techniques. This assessment model is then validated by the EA experts consist of the practitioners and academia. This assessment model was also tested by the former selected case studies and they were asked to give the feedback on its usability based on System Usability Survey (SUS) standard.

International Symposium on the $\quad 1 \quad$ London, U.K. Analytic Hierarchy Process 
ISAHP Article: A Style Guide for Paper Proposals To Be Submitted to the International Symposium on the Analytic Hierarchy Process 2016, London, U.K.

\section{Data/Model Analysis}

The EA implementation assessment model is built over six categories and 27 assessment criteria as shown in Figure 1 .

\begin{tabular}{|l|l|l|}
\hline INTERNAL \\
PROCESS
\end{tabular}

\begin{tabular}{|l|}
\hline AUTHORITY SUPPORT \\
\hline \\
\hline Stakeholder \\
Support \\
\hline Stakeholder Benefit \\
\hline Stakeholder \\
Recognition \\
\hline Mandate \\
\hline Political Influence \\
\hline Stakeholder \\
\hline
\end{tabular}

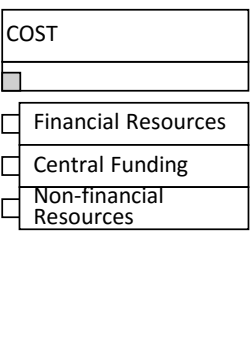

\begin{tabular}{|l|l|}
\hline TECHNOLOGY & TALENT \\
\hline MANAGEMENT \\
\hline EA Technology
\end{tabular}

Figure 1: Proposed categories and criteria for the EA implementation assessment model

Following the AHP methodology, the results from three case studies selected are presented in Table 1.

Table 1: EA implementation priority results from three case studies

\begin{tabular}{llrrr}
\hline CATEGORY & CRITERIA & CASE A & CASE B & CASE C \\
\hline INTERNAL & Business Driven Approach & $0.84 \%$ & $0.58 \%$ & $0.63 \%$ \\
PROCESS & Strategic Planning & $7.60 \%$ & $1.04 \%$ & $0.83 \%$ \\
& Implementation Roadmap & $0.47 \%$ & $1.47 \%$ & $1.01 \%$ \\
& Governance & $3.02 \%$ & $3.45 \%$ & $1.38 \%$ \\
& Rules \& Process & $1.06 \%$ & $2.36 \%$ & $2.39 \%$ \\
LEARNING AND & Organisation Value & $1.85 \%$ & $1.68 \%$ & $1.63 \%$ \\
GROWTH & Assessment & $11.12 \%$ & $0.59 \%$ & $0.90 \%$ \\
& Documentation & $1.58 \%$ & $1.42 \%$ & $1.02 \%$ \\
& Learning Culture & $5.96 \%$ & $1.49 \%$ & $1.48 \%$ \\
& Skill of Architect & $0.46 \%$ & $1.64 \%$ & $1.60 \%$ \\
& Training & $2.64 \%$ & $3.86 \%$ & $2.15 \%$ \\
AUTHORITY & Community of Practice & $1.41 \%$ & $2.80 \%$ & $2.79 \%$ \\
SUPPORT & Stakeholder Support & $20.76 \%$ & $1.29 \%$ & $0.89 \%$ \\
& Stakeholder Benefit & $13.24 \%$ & $1.45 \%$ & $1.29 \%$ \\
& EA Recognition & $1.98 \%$ & $3.43 \%$ & $1.55 \%$ \\
\hline
\end{tabular}

\begin{tabular}{llrrr}
\hline CATEGORY & CRITERIA & CASE A & CASE B & CASE C \\
\hline \multirow{5}{*}{ COST } & Mandate & $2.99 \%$ & $1.44 \%$ & $2.12 \%$ \\
& Political Influence & $2.64 \%$ & $1.46 \%$ & $2.70 \%$ \\
& Stakeholder Understanding & $3.84 \%$ & $3.38 \%$ & $4.14 \%$ \\
& Financial Resources & $6.41 \%$ & $6.40 \%$ & $2.69 \%$ \\
TECHNOLOGY & Non-financial Resources & $2.07 \%$ & $3.47 \%$ & $8.82 \%$ \\
& Central Funding & $0.87 \%$ & $4.71 \%$ & $5.77 \%$ \\
& Practical EA Technology & $3.68 \%$ & $6.27 \%$ & $3.71 \%$ \\
& EA Technology Support & $0.95 \%$ & $8.55 \%$ & $11.42 \%$ \\
TALENT & EA Repository & $0.60 \%$ & $6.58 \%$ & $6.54 \%$ \\
MANAGEMENT & Talent Management Plan & $1.59 \%$ & $13.12 \%$ & $4.89 \%$ \\
& Centralised Enterprise & $0.18 \%$ & $6.33 \%$ & $10.54 \%$ \\
& Architect & & & \\
& Retention Program & $0.18 \%$ & $9.77 \%$ & $15.13 \%$ \\
\hline
\end{tabular}

\section{Conclusions}

The formulation of this assessment model has provided a new perspective in EA implementation. This new model emphasises the importance of early assessment in any EA initiatives. This model also tailored to the organisation priority in EA implementation compared to existing generic models available. The evaluation results demonstrate that all selected case studies were satisfied with the assessment approach introduced and strongly recommended this assessment model to other organisations prior starting their EA initiative.

\section{Key References}

Bakar, N. A. A., Harihodin, S., and Kama, N. (2015). A Systematic Review of Enterprise Architecture Assessment Models. Paper presented at the Applied Mechanics and Materials, 339-343.

Pruijt, L., Slot, R., Plessius, H., Bos, R., and Brinkkemper, S. (2012). The Enterprise Architecture Realization Scorecard: A Result Oriented Assessment Instrument. In Trends in Enterprise Architecture Research and Practice-Driven Research on Enterprise Transformation (pp. 300-318): Springer.

Rodrigues, L. S., and Amaral, L. (2010). Enterprise Architecture Value Analysis Based on Value Drivers.

Sobczak, A. (2013). Methods of the Assessment of Enterprise Architecture Practice Maturity in an Organization. In Perspectives in Business Informatics Research (pp. 104-111): Springer.

Van der Raadt, B., Bonnet, M., Schouten, S., and Van Vliet, H. (2010). The relation between EA effectiveness and stakeholder satisfaction. Journal of Systems and Software, 83(10), 1954-1969. 